\title{
Capsule Commentary on Scales et al., Unintentional Continuation of Medications Intended for Acute Illness After Hospital Discharge: A Population-Based Cohort Study
}

\author{
Shoshana J. Herzig, MD, MPH \\ Division of General Medicine and Primary Care, Beth Israel Deaconess Medical Center, Boston, MA, USA.
}

J Gen Intern Med 31(2):224

DOI: $10.1007 / \mathrm{s} 11606-015-3543-8$

(C) Society of General Internal Medicine 2015

I $\mathrm{n}$ the present issue of JGIM, Scales et al. ${ }^{1}$ use a cohort of more than one million Canadian patients $>65$ years of age to identify rates and predictors of unintentional continuation of medications initiated during an acute hospitalization. Although the rates of unintentional continuation are relatively small, when considering the number of patients hospitalized annually, they translate into large numbers of patients with ongoing inadvertent exposure, and even larger costs, with an estimated total 1-year cost of over $\$ 18$ million.

Transitions in care are widely recognized as high-risk times for medication discrepancies, ${ }^{2,3}$ and part of medication reconciliation as advocated by the Institute for Healthcare Improvement includes ensuring that medications that are not appropriate for the next care setting are discontinued. ${ }^{4}$ The lack of information regarding the use of medication reconciliation tools by hospitals in this study renders us unable to distinguish between absence or failure of medication reconciliation. Despite this limitation, this study draws attention to the fact that current practices are suboptimal, particularly with respect to ensuring that inappropriate medications are discontinued, and that this type of medication error should be a focus of future innovative approaches to medication reconciliation.

Discontinuing medications that are no longer appropriate is a more complicated - and insufficiently addressed-issue than preventing omissions, ${ }^{5}$ as it requires understanding why the medication was started and what constitutes an appropriate indication for continuation. It is not surprising that longer hospitalizations are associated with unintentional continuation. There are multiple possible mechanisms involved, but one is that the indication for initial prescription is less likely to be known by the discharging provider the longer a patient is in the hospital. This finding suggests potential targets for improvement, such as requiring clinicians to document the reason for medication initiation in a way that is visible to the discharging provider, or enhanced pharmacist involvement for patients with long hospitalizations. Regardless of the approach, it is clear that standardized care processes, including enhanced clinical decision support, are necessary to ensure that we do not continue exposing our patients to unnecessary medications and harm after discharge.

Corresponding Author: Shoshana J. Herzig, MD, MPH; Division of General Medicine and Primary CareBeth Israel Deaconess Medical Center, Boston, MA 02446, USA (e-mail: sherzig@bidmc.harvard.edu).

\section{Compliance with Ethical Standards:}

Conflict of Interest: The author has no conflicts of interest with this article.

\section{REFERENCES}

1. Scales DC, Fischer HD, Li P, Bierman AS, Fernandes O, Mamdani M, Rochon P, Urbach DR, Bell CM. Unintentional Continuation of Medications Intended for Acute Illness After Hospital Discharge: A population-based cohort study. J Gen Intern Med. doi:10.1007/s11606-015-3501-5.

2. Coleman EA, Smith JD, Raha D, Min SJ. Posthospital medication discrepancies: prevalence and contributing factors. Arch Intern Med. 2005; 165(16): 1842-7.

3. Rozich JD, Howard RJ, Justeson JM, Macken PD, Lindsay ME, Resar RK. Standardization as a mechanism to improve safety in health care. Jt Comm J Qual Saf. 2004;30(1):5-14.

4. Institute for Healthcare Improvement. Reconcile Medications At All Transition Points. http://www.ihi.org/resources/Pages/Changes/ ReconcileMedicationsatAllTransitionPoints.aspx. Accessed September 8, 2015

5. Zeigler AJ, McAllen KJ, Slot MG, Barletta JF. Medication reconciliation effect on prolonged inpatient stress ulcer prophylaxis. Ann Pharmacother. 2008;42(7):940-6. 